\title{
BEAM DYNAMICS SIMULATIONS FOR LINACS DRIVING SHORT-WAVELENGTH FELS
}

\author{
M. Ferrario, F. Tazzioli, LNF-INFN, Frascati \\ L. Serafini, Univ. of Milan and INFN-Milan
}

\begin{abstract}
The fast code HOMDYN has been recently developed, in the framework of the TTF (TESLA Test Facility) collaboration, in order to study the beam dynamics of linacs delivering high brightness beams as those needed for short wavelength FEL experiments. These linacs are typically driven by radio-frequency photo-injectors, where correlated time dependent space charge effects are of great relevance: these effects cannot be studied by standard beam optics codes (TRACE3D, etc.) and they have been modeled so far by means of multi-particle (PIC or quasistatic) codes requiring heavy cpu time and memory allocations. HOMDYN is able to describe the beam generation at the photo-cathode and the emittance compensation process in the injector even running on a laptop with very modest running times (less than a minute). In this paper we show how this capability of the code is exploited so to model a whole linac up to the point where the space charge dominated regime is of relevance $(200 \mathrm{MeV})$.
\end{abstract}

\section{GENERAL CRITERIA FOR OPTIMIZATION OF A PHOTO-INJECTOR}

The art of designing optimized RF Photo-Injectors capable to deliver high brightness electron beams has moved in the last decade from a cut and try procedure, guided by rule of the thumb guesses and going through time consuming simulations, up to a fast parameter space scanning guided by the analytical results of the theoretical model for laminar beams [1], achieved by means of a fast running code based on a multi-beam multi-envelope description of the beam dynamics. By this technique it is possible to study the time dependent space charge problem inherent in the beam dynamics of such devices, so to reach the optimum operating point which corresponds to maximum beam brightness.

The code HOMDYN [2] is actually tailored to describe, within a multi-envelope multi-beam frame, the space charge dominated dynamics of laminar beams in presence of correlated, or time dependent space charge forces: because it's not a multi-particle code, but a multienvelope one, the code behaves like any beam transport code like TRACE-3D or TRANSPORT, giving rise to very fast modeling capability for photo-injectors.

It has been by now understood that the optimization of a photo-injector corresponds to accelerating and propagating the beam through the device as close as possible to two beam equilibrium, a laminar Brillouin flow (in drifts) and the so-called invariant envelope (in accelerating sections) which is a generalization of Brillouin flow for an accelerated beam. In this case the beam undergoes cold plasma oscillations, i.e. the space charge collective force is largely dominant over the emittance pressure, where the betatron motion (trajectory cross-over) is almost absent (laminar flow) and the frequency of the plasma oscillations due to mismatches between the space charge force and the external focusing gradient is to first order independent on the current. It is such a frequency independence that brings to reversible normalized emittance oscillations: accelerating the beam through the invariant envelope just makes these oscillations damped like the square root of the beam energy, bringing the normalized emittance at the injector exit down to a steady state minimum when the oscillations are properly tuned.

The laminar behavior of an electron beam is characterized by the laminarity parameter

$$
\rho \equiv\left\{\frac{\left[\operatorname{Ig}(\zeta) / 2 I_{0}\right]}{\varepsilon_{n, t h} \gamma \sqrt{3 \gamma^{\prime 2} / 4+\Omega_{L}^{2}}}\right\}^{2}
$$

which is defined in terms of the beam average energy $\gamma$, the peak current $I\left(I_{0}=17 \mathrm{kA}\right)$, the accelerating gradient $\gamma^{\prime}=e E_{a c c} / m c^{2}$, the Larmor frequency of the solenoid focusing field $\Omega=\frac{e B_{S O L}}{2 m}$ and the rms normalized thermal emittance $\varepsilon_{n, t h}$. The beam is said to be laminar whenever

$\rho>>1$, which occurs from the photo-cathode surface up to energies even in excess of $100 \mathrm{MeV}$ for beams carrying about $100 \mathrm{~A}$ of peak current.

The time dependence of the space charge field effects is expressed through the geometrical factor $g(\zeta)$, which is a function of the longitudinal position in the electron bunch (the so-called slice position), defined as $\zeta \equiv z-\beta c t+z_{0}$.

Whenever the geometrical factor $g(\zeta)$ has a significant variation along the bunch, we obtain a beam perveance term in the rms envelope equation, which is slicedependent, therefore we are in presence of time (or slice) dependent effects. In the case of a cylindrical bunch we have for instance

$$
g \cong 1-\frac{2 A^{2}}{\gamma^{2}}\left[1+12\left(\frac{\zeta}{L}\right)^{2}+80\left(\frac{\zeta}{L}\right)^{4}\right]
$$


showing that when the bunch aspect ratio $A \equiv R / L$ is not much smaller than 1 the dependence on the slice coordinate can be quite relevant.

It has been shown that the rms projected normalized emittance $\varepsilon_{n} \equiv p \sqrt{\left\langle x^{2}\right\rangle\left\langle x^{\prime 2}\right\rangle-\left\langle x x^{\prime}\right\rangle^{2}}$ oscillates with a frequency $\sqrt{2 K_{r}}$ at an amplitude $\Delta \varepsilon_{n} \propto \sqrt{\frac{I\langle g\rangle}{\gamma}} \sqrt{\left\langle g^{2}\right\rangle /\langle g\rangle^{2}-1}$ whenever a bunched beam (i.e. $g(\zeta) \neq 1$ ) is rms matched into a focusing channel of gradient $K_{r}$, i.e. on a Brillouin flow equilibrium $\sigma_{B}(\zeta)=\sqrt{\frac{I g(\zeta)}{2 I_{0} \gamma^{3} K_{r}}}$ (where $\sigma$ is the rms beam spot size, eventually slice dependent). Accelerating on the invariant envelope $\sigma_{i n v}(\zeta)=\frac{2}{\gamma^{\prime}} \sqrt{\frac{I g(\zeta)}{3 I_{0} \gamma}}$, which is a particular exact solution of the rms envelope equation in the laminar flow regime, brings the emittance to damped oscillations. The basic point in the design of a photoinjector is therefore to match properly the beam at injection into any accelerating section, according to these criteria:

$\hat{\sigma}^{\prime}=0$, implying a laminar waist at injection

$\gamma^{\prime}=\frac{2}{\sigma_{w}} \sqrt{\frac{I\langle g\rangle}{3 I_{0} \gamma}}$, i.e. an rms match on the inv. env.

These also requires that focusing elements in drifts be spaced close to one quarter of plasma oscillation wavelength, given by $\lambda_{p}=\frac{4 \pi \gamma}{\gamma^{\prime}} \sqrt{\frac{2}{3}}$.

\section{APPLICATION TO THE TTF-FEL LINAC}

In this section we study how to implement these matching criteria into the actual design of the TTF linac. up to the end of the second cryomodule, corresponding to a beam energy of $200 \mathrm{MeV}$. A previous analysis [3] was devoted to a general lay-out for the linac with complete degree of freedom in the spacing of focusing elements and accelerating sections (i.e. the cryostats containing $8 \mathrm{SC}$ accelerating cavities, each with 9 cells working at 1.3 $\mathrm{GHz}$ ). In the following we will compare those results to the best one can do with the actual lay-out neglecting compressor magnets, not yet implemented in to the code. The $1 \mathrm{nC}$ beam is generated in one and half cells RF-gun operating at $1.3 \mathrm{GHz}$ with $40 \mathrm{MV} / \mathrm{m}$ peak field on the cathode, by a 8 ps (sigma) long laser pulse with $1.5 \mathrm{~mm}$ radius. The gun is embedded in a $0.11 \mathrm{~T}$ split solenoid field. In the drifting tube downstream the gun the rms emittance reach a first minimum $(0.9 \mathrm{~mm} \mathrm{mrad})$ corresponding to the minimum $\left(\sigma^{\prime}=0\right)$ envelope $\left(\sigma_{\mathrm{r}}=1.18\right.$ $\mathrm{mm})$ at $\mathrm{z}=1.2 \mathrm{~m}$ from the cathode, where the booster is placed,(Fig. 1).

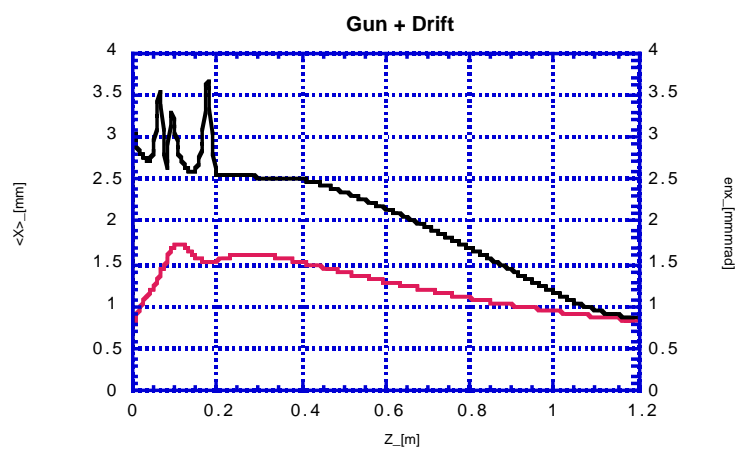

Figure 1: Beam envelope and rms normalized emittance in the gun and drift up to the booster entrance.

To match the booster cavity ( 9 cells $1.3 \mathrm{GHz}$ superconducting cavity) to the beam we use the previous criteria to compute the accelerating field needed. It results to be $10 \mathrm{MV} / \mathrm{m}$ with $\mathrm{I}=40 \mathrm{~A}$ and $\gamma=10$.

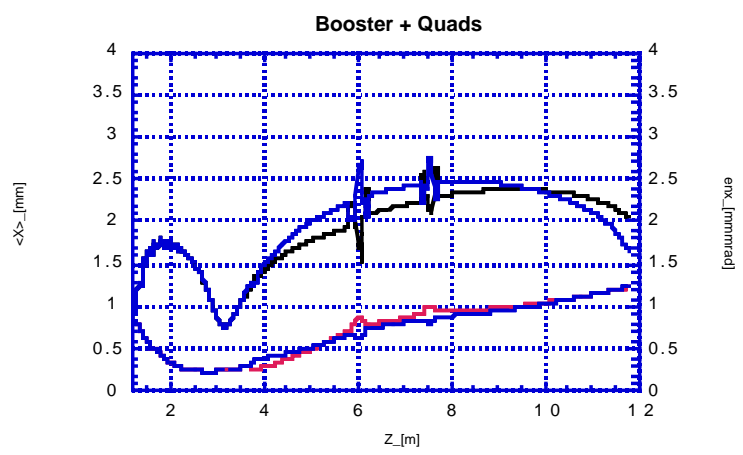

Figure 2: Beam envelope and rms normalized emittance in the booster and drift up to the cryomodule 1 entrance.

Downstream the booster the rms emittance reach a second minimum $(0.8 \mathrm{~mm} \mathrm{mrad})$ in the drift and at the booster exit $(\mathrm{z}=2.4 \mathrm{~m})$ the beam envelope $\left(\sigma_{\mathrm{r}}=0.35 \mathrm{~mm}\right)$ is still parallel to the $\mathrm{z}$-axis with $\gamma=35$. In the ideal case, as discussed in [3], the envelope should be allowed to perform small oscillations around the equilibrium value reached in the booster and smoothly transported to match the condition $\sigma^{\prime}=0$ at the entrance of the first cryo-module. The situation is here complicated by the technical constrains of the drift section, foreseen to house focusing elements (one quadrupole doublet and two triplets), diagnostic stations and a chicane compressor. In addition by using quadrupoles as focusing elements the envelope and the emittance oscillations are split in the $\mathrm{x}$ and $\mathrm{y}$ planes, resulting in a difficult task to match both planes to the cryo-module. 


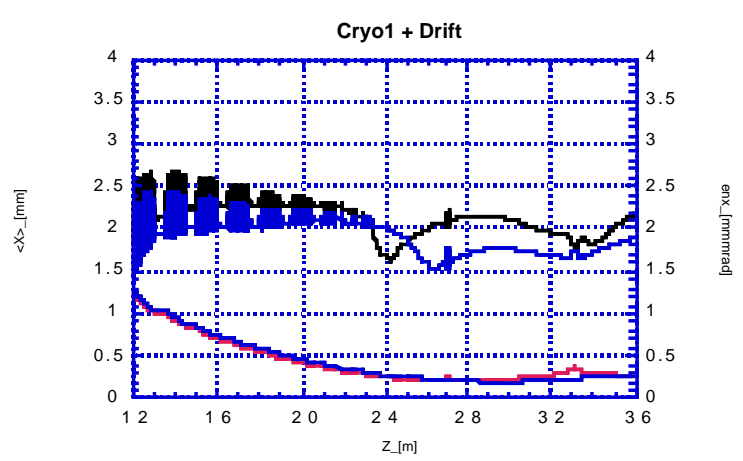

Figure 3: Beam envelope and rms normalized emittance in cryomodule 1 and drift up to the cryomodule 2 entrance.

The best solution we found results to be the less perturbing to the cylindrical symmetry of the beam (Fig. 2). We allowed indeed the envelope to diverge up to $\sigma_{\mathrm{r}}=$ $1.75 \mathrm{~mm}$ with low quadruoles gradients so that to keep the emittance oscillation in phase in both planes up to the entrance of the first cryomodule $(\mathrm{z}=11.7 \mathrm{~m})$. The beam is there boosted with $11 \mathrm{MV} / \mathrm{m}$ accelerating field, a low gradient is chosen to avoid over focussing in the accelerating section. Emittance oscillations are damped during acceleration around $2 \mathrm{~mm}$ mrad (Fig. 3).

With the same care the beam is transported to the second cryomodule through a quadrupole focusing channel and boosted up to $200 \mathrm{MeV}$ in the second cryomodule (Fig. $4)$.

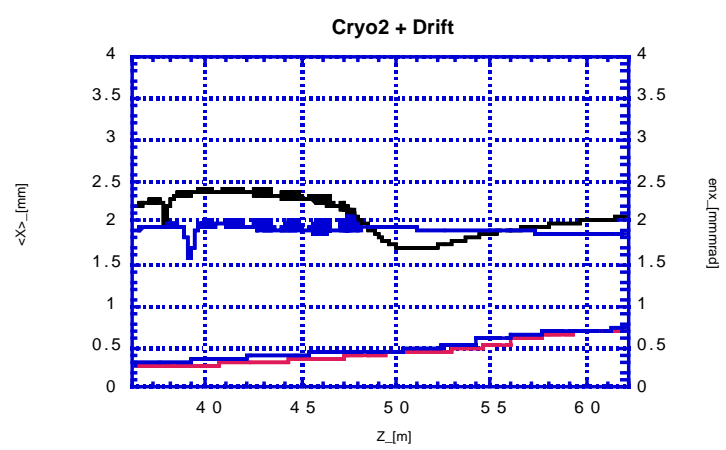

Figure 4: Beam envelope and rms normalized emittance in cryomodule 2 and drift.

\section{CONCLUSIONS}

By joining the analytical predictions and optimizing criteria produced by the theory of intense relativistic laminar beams with the powerful capability of the new code HOMDYN to model a full photoinjector up to full scale energy $(200 \mathrm{MeV})$ in very short cpu times (minutes on a laptop), the optimization of a new design or the search for the optimum operating point of a present layout can be accomplished quickly and easily. We believe that the general design of future injectors will benefit from such a new user-friend procedure.

\section{REFERENCES}

[1] L. Serafini, J. B. Rosenzweig, "Envelope analysis of intense relativistic quasilaminar beams in $\mathrm{rf}$ photoinjectors: a theory of emittance compensation", Phys. Rev. E, 55, (1997)

[2] M. Ferrario,.A. Mosnier, L. Serafini, F. Tazzioli, J. M. Tessier, "Multi-bunch energy spread induced by beam loading in a standing wave structure", Part. Acc., 52 (1996)

[3] M. Ferrario, L. Serafini, "Multi-bunch dynamics in $\mathrm{rf}$ photoinjectors through an envelope equation approach', Proc. of VI EPAC, Stockholm (1998). 